\title{
APRENDIZADO CENTRADO EM PROBLEMAS
}

\author{
PROBLEM-BASED LEARNING
}

Maria de Lourdes Veronese Rodrigues \& José Fernando de Castro Figueiredo

Docentes dos Departamentos: Oftalmologia e Otorrinolaringologia; Clínica Médica (Divisão de Moléstias Infecciosas e Tropicais); Membros da Comissão de Graduação; Faculdade de Medicina de Ribeirão Preto da Universidade de São Paulo.

CorRespondêncIa: Prof.Dra. Maria de Lourdes V. Rodrigues - Departamento de Oftalmologia e Otorrinolaringologia da Faculdade de Medicina de Ribeirão Preto - Campus Universitário - CEP: 14048-900 - Ribeirão Preto - SP — mdlvrodr@fmrp.usp.br.

RODRIGUES MLV \& FIGUEIREDO JFC. Aprendizado centrado em problemas. Medicina, Ribeirão Preto, 29: 396-402, out./dez. 1996.

RESUMO: Aprendizado Centrado em Problemas (PBL) é metodologia de ensino na qual os tópicos de aprendizado são identificados a partir da apresentação de um problema real ou simulado. Frente ao problema o estudante desenvolve meios para solucioná-lo e neste processo identifica lacunas em seu conhecimento, buscando preenchê-las, com o auxílio de recursos instrucionais disponíveis. Essa metodologia tem como características principais desenvolver no estudante as habilidades de gerenciar o próprio aprendizado, de integrar conhecimentos e de identificar e explorar áreas novas.

Esse método é idealmente aplicável ao aprendizado auto-dirigível centrado no aluno. O sucesso da utilização da metodologia PBL depende de uma série de pré-requisitos e esses podem influenciar na forma de implantação e na exeqüibilidade do método.

Como exemplos de necessidades para a implantação do método PBL podemos citar, dentre outras: necessidade de espaço livre para o auto-aprendizado do aluno; necessidade de revisão do papel do docente no processo de ensino-aprendizagem; revisão do conceito de autonomia departamental; mudanças dos critérios de seleção dos alunos; e investimentos financeiros em infra-estrutura.

Algumas faculdades de Medicina o utilizam de forma exclusiva na formação de seus alunos, outras somente para grupos de alunos e outras, ainda, em segmentos do currículo tradicional.

Desta forma, seria recomendável que essa metodologia, se cogitada a sua aplicação na Faculdade de Medicina de Ribeirão Preto, se restringisse, no início, a determinadas unidades de ensino ou segmentos da estrutura curricular vigente. A avaliação criteriosa dos resultados determinaria a conveniência da utilização mais ampla do método.

UNITERMOS: Educação Médica. Aprendizado. Faculdade de Medicina.

\section{INTRODUÇÃO}

O ensino da Medicina pode ser, de forma genérica, desenvolvido de duas maneiras. A primeira delas diz respeito a quem deva ser o principal responsável pelo processo de ensino/aprendizagem (professor ou o aluno) e a outra, à forma como o conteúdo a ser ensinado deva ser organizado (centrado em assunto/temas ou na solução de problemas).

Dessa forma, o ensino pode ser caracterizado em quatro modalidades: centrado no professor, baseado em assuntos/temas; centrado no estudante, baseado em assuntos/temas; centrado no professor, baseado na solução de problemas; centrado no estudante, baseado na solução de problemas. 
O ensino tradicional da Medicina, desenvolvi do através da ministração de disciplinas por "experts", baseado em aulas formais, seminários e aulas práticas, caracteriza o ensino centrado no professor e baseado em assuntos/temas.

Como vantagens desse método, temos que os professores, geralmente, são especialistas que conhecem em profundidade o assunto que ministram, o que os leva a identificar facilmente os níveis de conhecimento prévio necessários para a compreensão de um determinado tema. Isso facilita a composição do programa do curso e auxilia o aluno na busca do conhecimento. Além disso, nesse sistema, os limites do aprendizado são melhor definidos, os recursos instrucionais mais facilmente identificáveis e é dado ao professor um controle maior sobre os conceitos e habilidades que necessitam ser aprendidos. Por outro lado, ao centralizar no professor a responsabilidade de gerenciar todo o processo de ensino/aprendizagem, esse método traz desvantagens. Como exemplo, podem ocorrer distorções no conteúdo programático, em função do grau de especialização do professor. Além disso, muitas vezes, os professores responsáveis pelos programas de ensino têm também outras atividades, como as clínico-cirúrgicas, pesquisa e administração, sobrando menos tempo para a dedicação ao ensino. Acresce-se a isso o fato de que as escolas médicas, de modo geral, não têm valorizado adequadamente as atividades relacionadas ao ensino de graduação e esse item tem pouco peso como fator de progressão na carreira, causa de desmotivação para a atividade docente.

Outras desvantagens a serem consideradas dizem respeito aos estudantes. Estes não são uniformes quanto a sua bagagem de conhecimento prévio e em relação ao seu ritmo e estilo de aprendizado, além de, freqüentemente, terem aspirações profissionais diversas. Assim, o professor, ao assumir o gerenciamento pleno do processo, não tem como controlar o aprendizado individual.

Essa metodologia resulta em um aprendizado por memorização de conceitos, que podem ser rapidamente perdidos, já ao longo do curso. Uma desvantagem adicional deriva do fato de que, nessa forma de ensino, a integração de conhecimentos e habilidades é dificultada, uma vez que as informações são absorvidas de maneira dissociada da prática imediata, sem um foco ao redor do qual se organize o conhecimento. Essa desvantagem deve ser particularmente ressalta da, urna vez que o exercício da prática médica é fundamentado na integração de conceitos e habilidades.
Algumas dessas desvantagens, acrescidas à outras necessidades de reforma, foram a razão pela qual, em meados da década de sessenta, se propôs para a área médica uma forma de ensino centrada no estudante e baseada na solução de problemas ("Problem Based Learning" - PBL), adaptada de experiências já em andamento em outras áreas do conhecimento.

\section{APRENDIZADO CENTRADO EM PROBLEMAS}

Aprendizado centrado em problemas é metodologia de ensino na qual os tópicos a serem aprendi dos são identificados a partir da apresentação de um problema real ou simulado a um grupo de alunos. Para solucionar este problema é necessário recorrer aos conhecimentos prévios, adquirir novos conhecimentos e integrá-los. Essa integração, aliada à aplicação prática imediata, facilita a retenção do conhecimento, que pode ser mais facilmente resgatado, quando o estudante estiver diante de novos problemas.

A base filosófica que norteou a proposição desse modelo de aprendizagem foi a consciência de que o aprendizado do ser humano se faz a partir de experiências do seu cotidiano - aprender resolvendo problemas'. Ao ser apresentado a um problema novo, o estudante se vê na contingência de ter que desenvolver meios para solucioná-lo. Através da obtenção, análise e síntese dos dados disponíveis, ele identifica lacunas do conhecimento, buscando preenchê-las. A aplicação desses novos conhecimentos, em conjunto com métodos de raciocínio dedutivo, compõe as bases para a solução do problema em foco. Esse tipo de abordagem leva o estudante a "aprender a aprender"1.

Essa metodologia tem corno principais objetivos desenvolver no estudante as habilidades de gerenciar o próprio aprendizado, de integrar o conhecimento, de identificar e explorar áreas novas. Essas habilidades são fundamentais para a prática médica, pois esta exige integração de conhecimentos e a Medicina, por ser ciência dinâmica, exige do médico educação continuada ao longo de sua vida profissional.

Esse método é idealmente aplicável ao aprendizado auto-dirigido, centrado no aluno.

No entanto, o sucesso da utilização da metodologia PBL depende de uma série de pré-requisitos: os estudantes devem ter características de personalidade adequadas (independência, determinação, senso de responsabilidade, capacidade de comunicação, 
desinibição, capacidade de organização); o corpo docente deve ser treinado e familiarizado com o método; na organização da estrutura curricular, deve ser previsto tempo adequado para o estudo auto-dirigido; a instituição deve dispor da infra-estrutura necessária para o auto-aprendizado do aluno (material instrucional adequado e adaptado para o método, salas para reuniões de pequenos grupos, bibliotecas, laboratórios, recursos audio-visuais).

Um exemplo de esquema da aplicação do método, utilizado na Harvard Medical School, é apresentado no esquema abaixo ${ }^{2}$ :

\section{Fase 1:}

- Identificação do(s) problema(s)

- Formulação de Hipóteses

- Solicitação de Dados Adicionais

- Identificação de Temas de Aprendizado

- Elaboração do Cronograma de Aprendizado

Estudo Independente

\section{Fase II:}

- Retorno ao Problema

- Crítica e Aplicação das Novas Informações

- Solicitação de Dados Adicionais

- Redefinição do Problema

- Reformulação de Hipóteses

- Identificação de novos Temas de Aprendizado

- Anotação das Fontes

Fase III:

- Retorno ao Processo

- Síntese do Aprendizado

- Avaliação.

Em cada uma dessas fases, pequenos grupos de alunos (idealmente de 5 a 8) são acompanhados por tutores que, a partir de objetivos educacionais previamente definidos, orientam o acesso do estudante ao seu conhecimento prévio e o ajudam a detectar suas limitações deste conhecimento. O grupo de alunos, identificando a sua deficiência sobre assuntos relevantes para a compreensão e solução do problema apresentado, divide as tarefas na busca de fontes disponíveis na instituição (laboratórios, bibliotecas, centros de processamento de dados, etc.) e os alunos retornam ao problema. Esse processo ("tutorial") se repete quantas vezes sejam necessárias para que os estudantes, articulando os conhecimentos obtidos com os da dos disponíveis, solucionem o problema em questão. A apresentação de novos problemas, ao longo de um período definido (usualmente 4 a 6 semanas), faz com que os conteúdos instrucionais pré-estabelecidos sejam adequadamente cumpridos de forma integrada ${ }^{1,3}$.

\section{FORMAS DE IMPLANTAÇÃO DO MÉTODO}

A implantação do método PBL requer condições peculiares e as particularidades das instituições (infra-estrutura, características do corpo docente, metodologia de seleção dos ingressantes, etc.) influenciam a sua exeqüibilidade e modo de implantação. Um critério adicional, que deve ser analisado, diz respeito aos custos financeiros exigidos para a adequação da instituição à metodologia.

Após a implantação desse método na McMaster University, diversas escolas de Medicina passaram a utilizá-lo, quer seja de forma plena, quer seja através da criação de currículos paralelos ou ainda em segmentos da grade curricular. A forma de iniciar a metodologia de PBL tem diferido de uma instituição para outra. Algumas já se iniciaram com esta metodologia (ex: Mc Master University), outras, tais como a Southern Illinois School of Medicine introduziram um currículo PBL em paralelo ao currículo tradicional, mantendo os dois tipos de curso. Outras, ainda, substituiíram o currículo tradicional pelo PBL, sem um período de transição (Faculté de Medicine - Université de Sherbrooke); outras o iniciaram com um subgrupo de alunos, paralelamente ao currículo tradicional, que foi gradualmente substituído pelo currículo PBL (Harvard Medical School); outras iniciaram as mudanças com um currículo paralelo em PBL e posteriormente o reduziram para um segmento do currículo tradicional (Faculdade de Medicina - Chulalongkorn University $)^{4 / 9}$.

\section{EXEMPLOS DE ESTRUTURAS CUR- RICULARES QUE UTILIZAM METO- DOLOGIA DE PBL(*)}

\footnotetext{
- Faculté de Medicine - Université de Sherbrooke, Sherbrooke, Quebec, Canadá

Quando os primeiros estudantes foram admiti dos, em 1966, esta Faculdade já havia adotado um currículo inovador: acompanhando o modelo da Case Western Reserve University, era oferecido um programa pré-clínico, baseado na integração de assuntos e disciplinas, em um modelo curricular centrado em órgãos e sistemas. Dessa forma, os professores da faculdade passaram a ter, a partir dessa época, famili-
}

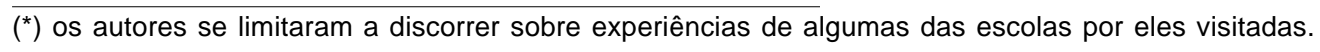


aridade com o ensino interdisciplinar, administrado de forma interdepartamental, requerido pelos programas baseados em órgãos e sistemas. No entanto, algumas características da educação médica tradicional (conteúdo excessivo, aprendizado centrado no professor, integração inadequada entre ciências básicas e ciências clínicas) motivaram, a partir de 1987, uma revi são nos programas, com a mudança completa do sistema tradicional para um programa de PBL. Esse programa é desenvolvido, de forma resumida, da seguinte maneira: o curso tem a duração total de 4 anos; o período pré-clínico é dividido em três fases: a Fase 1, com duração total de 15 semanas inclui urna Introdução à Medicina e aos Métodos de Aprendizagem (2 semanas), seguida de um período de II semanas de Introdução aos Conceitos Básicos de Biologia Médica, utilizando metodologia de PBL. A Fase 1 se encerra após um período de 3 semanas em hospitais comunitários da província de Quebec. A Fase II, com duração de 18 meses, capacita o estudante a integrar ciências básicas com ciências clínicas, através de uma sequiência de 15 unidades de PBL, com duração de 3 a 6 semanas cada uma. Essas unidades são dividi das de acordo com órgãos e sistemas mais relevantes do corpo humano e sua seqüência é a seguinte: Crescimento, Desenvolvimento e Envelhecimento, Sistema Nervoso, Ciências Psíquicas, Aparelho Locomotor, Saúde Comunitária, Aparelho Cardiovascular, Aparelho Respiratório, Aparelho Digestivo, Apare lho Urinário, Sistema Hematológico e Imunológico, Doenças Infecciosas, Estágio de PBL em Comunidade, Sistema Endócrino, Aparelho Reprodutor e Sexualidade Humana.

A Fase III compreende uma unidade de integração pré-internato, utilizando método específico de PBL para atividades centradas em interdisciplinaridade, em Ética Médica e em Medicina Legal. Segue-se a ela o Internato, com duração de 42 semanas de estágios rotatórios compulsórios nas disciplinas clínicas mais relevantes (Medicina Interna, Obstetrícia e Ginecologia, Pediatria, Psiquiatria e Cirurgia), aos quais se adicionam 7 semanas de estágio rotatório na comunidade (Medicina de Família, Primeiros Socorros e Medicina Comunitária). Os alunos desenvolvem, também nesta etapa, estágios rotatórios optativos com duração de 12 semanas. No final do período de Internato, os estudantes dispõem de seis semanas para o preparo individual para os exames nacionais de capacitação. Ao longo do programa, os alunos são avalia dos em diversos momentos, com avaliações somativas no final das unidades e no final das diferentes etapas do curso. Essa avaliação é feita de forma sistematiza da pelos tutores de cada grupo, a qual se soma a avaliação dos pares e do próprio aluno ${ }^{4,7,8}$.

\section{- Harvard Medical School, Boston, Massachusetts, Estados Unidos da América}

O método de PBL foi iniciado na Harvard Medical School, em 1985, como um "new pathway", paralelo ao currículo tradicional e oferecido a 24 alunos, que passaram a compor a "Oliver Wendell Holmes Society". Como a experiência foi bem sucedida, em 1987, todo o corpo discente foi incorporado na nova estrutura curricular, passando a constituir mais três societies (Cannon, Castle e Peabody) com aproximadamente 40 alunos cada uma. Um docente senior passou a atuar como "master" em cada sociedade e foram criados espaços apropriados para que alunos e professores pudessem se encontrar, estudar e para atividades de lazer. Foi designado, a partir dessa época, um assistente administrativo, em tempo integral, para assessorar o funcionamento do dia a dia de cada "society". Essa estrutura curricular floresceu sendo agora conhecida como "Program in Medical Education" (PME).

$\mathrm{O}$ curso se desenvolve em quatro anos. No primeiro ano, são oferecidos três blocos de disciplinas:

a) Biologia Humana, composto por Corpo Humano (Anatomia Macroscópica, Histologia, Radiologia e Biologia Celular), com duração de 8 semanas; Metabolismo e Função dos Órgãos e Sistemas Humanos (Bioquímica, Fisiologia, Biofísica e Biologia Molecular), com duração de oito semanas; Farmacologia, com duração de quatro semanas: Genética, Embriologia e Reprodução (Genética Humana e Molecular, Embriologia e Morfogênese e Reprodução), com duração de seis semanas, e Identidade, Micróbios e Defesa (Imunologia, Microbiologia e Patologia), com duração de 10 semanas;

b) Patient-Doctor 1, ministrada em 34 sessões (duas horas por semana) e

c) Medicina Social e Bioestatístical Epidemiologia (duas horas por semana).

No segundo ano, são ministrados os seguintes blocos de disciplinas:

a) Biologia Humana, composto por Sistema Nervoso e Comportamento (Neurologia, Psiquiatria e Neuroanatomia, Neurofisiologia, Neurofisiopatologia), com duração de 10 semanas e Sistemas Humanos (Fisiopatologia dos principais Sistemas Orgânicos), com duração total de 33 semanas; 
b) Patient-Doctor II (Introdução à Medicina Clínica) ministrado em 36 sessões (duas horas por semana);

c) Psicopatologia (três horas por semana) e Medicina Preventiva (duas horas por semana).

As unidades de ensino ministradas no terceiro ano são:

a) Patient-Doctor III (Introdução ao Ensino Hospitalar, Política Financeira em Saúde, Conduta nas Situações de Risco, Ética, Serviço Social, visita à Hospitais Psiquiátricos, Escolha da Carreira, a Morte e o Morrer, Dificuldades do Paciente, Relações da Etnia e da Cultura com a Doença, Projetos de Grupo, Geriatria e Humanidades) ministrado em 46 tutorials;

b) início do Internato, que continua no $4^{\circ}$ ano e compreende estágios rotatórios nas seguintes áreas: Medicina Interna, Prática em Ambulatório, Saúde da Mulher e da Criança, Cirurgia, Neurologia, Psiquiatria e Radiologia. No quarto ano, os alunos fazem ainda um estágio denominado "Senior Clinical Electives" e curso optativo que pode ser escolhido entre dezessete que são oferecidos ${ }^{2,6,8,10}$.

\section{- Faculty of Medicine - Chulalongkorn University, Bangkok, Tailândia}

Em razão das mudanças ocorridas na Tailândia nas últimas décadas, foram tomadas decisões, no sentido de modificar a tendência de concentração dos médicos nos grandes centros urbanos do país. Em decorrência disso, optou-se pela formação de diferentes tipos de médicos. Ao lado do curso tradicional, a partir de 1978, uma parte dos alunos foi alocada no "Projeto de Educação Médica para Estudantes Oriundos de Áreas Rurais (MESRAP)", ambos com aprendizado centrado no professor. Posteriormente, a partir de 1988, foi incorporado um terceiro modelo, em paralelo com os anteriores, baseado em metodologia PBL. Esses programas se desenvolveram até 1993. Em razão da exigência de grandes investimentos em recursos humanos e materiais para a manutenção dos três projetos, a universidade optou por fundi-los em um único programa ("combined track"), que pretende reunir as vantagens de cada um deles, e que passou a ser adotado a partir de 1994.

$\mathrm{O}$ "combined track" tem seu primeiro ano realizado no Centro de Ciências da Chulalongkorn University, compreendendo as seguintes disciplinas: Fundamentos de Inglês, Estatística para as Ciências Biológicas, Cálculo 1, Laboratório de Química Geral, Química Orgânica, Laboratório de Química Orgânica, Biologia Geral, Laboratório de Biologia Geral, Física Médica, Laboratório de Física Médica, Microbio- logia Geral, Ciência do Comportamento, Sociedade e Cultura.

O segundo e o terceiro anos, com duração de quatro semestres, constituem o período pré-clínico. $\mathrm{O}$ primeiro semestre do pré-clínico é desenvolvido na Faculdade de Medicina da Chulalongkorn University e compreende oito disciplinas, que se agregam num conjunto maior denominado Estrutura Humana e Funções, ministradas de maneira interdisciplinar, com participação de diversos departamentos. No segundo semestre, são ministradas quatro semanas de Metabolismo e Nutrição; duas semanas de Introdução à Saúde e à Doença; cinco semanas de Reprodução; cinco semanas de Crescimento e Desenvolvimento, duas semanas de Patologia Básica e duas semanas de Optativos em Medicina Comunitária. Nesse momento, a partir do módulo Introdução à Saúde e à Doença se inicia a metodologia de PBL, que se estende até o final do período pré-clínico.

O primeiro semestre do terceiro ano é composto por seis semanas de Neurociências; duas semanas de imunologia Básica e dez semanas de Infecção, compreendendo cinco disciplinas (Microbiologia, Parasitologia, Patologia, Farmacologia e Medicina Laboratorial). O segundo semestre do terceiro ano compreende uma semana de Imunologia Clínica; duas semanas de Patologia Clínica; quatro semanas de Oncologia; quatro semanas de Doenças Degenerativas; duas semanas de Epidemiologia e Bioestatística; duas semanas de Saúde Ambiental e Ocupacional; três semanas de Desenvolvimento Humano e duas semanas de estágio optativo em Medicina Comunitária.

O quarto, quinto e sexto anos, compõem o curso clínico. O quarto ano é constituído por duas semanas de Introdução à Clínica; uma semana de Medicina Comunitária; doze semanas de Clínica Médica; doze semanas de Cirurgia; doze semanas de Ginecologia e Obstetrícia; uma semana de Ortopedia; uma semana de Medicina Legal; uma semana de Oftalmologia e uma semana de Otorrinolaringologia.

No quinto ano, são ministradas seis semanas de Cirurgia; doze semanas de Clínica Médica; três semanas de Otorrinolaringologia; três semanas de Oftalmologia; doze semanas de Pediatria; duas semanas de Ortopedia; duas semanas de Medicina Física e Reabilitação; quatro semanas de Radiologia; quatro semanas de Psiquiatria e duas semanas de Medicina Legal.

O sexto ano corresponde a oito semanas de Clínica Médica; quatro semanas de Emergências Médicas; quatro semanas de Medicina Comunitária; quatro semanas de Anestesiologia; quatro semanas de Prática Médica Geral; seis semanas de Pediatria; seis 
semanas de Ginecologia/Obstetrícia; nove se manas de Cirurgia; três semanas de Ortopedia e duas semanas de Optativos. Durante o sexto ano os alunos desenvolvem suas atividades, além de no Chulalongkorn Hospital, também em hospitais provinciais (Bhurnipol Hospital, Chantaburi Hospital e Chonburi Hospital).

Ultrapassada esta fase, os alunos são submetidos ao exame final, para obtenção do grau de médico ${ }^{5}$

\section{CONSIDERAÇÕES FINAIS}

A metodologia de PBL tem se mostrado um instrumento valioso na formação do médico, com vantagens sobre o método de ensino tradicional.

No entanto, para a sua implantação há necessidade de um considerável esforço institucional (necessidade de espaço livre na estrutura curricular para o auto-aprendizado do aluno, necessidade, por parte dos docentes, de realizar trabalho integrado e em equipe, necessidade de mudança na mentalidade sobre o papel do docente no processo ensino-aprendizagem, revisão do conceito de autonomia departamental, mudança no critério de seleção dos alunos, mudança na forma de avaliação durante o curso, necessidade de formação técnica de recursos humanos para a metodologia PBL, investimentos financeiros em infraestrutura, tais como elaboração de casos clínicos, outros recursos instrucionais, reformas e construção para adaptação do ambiente físico, etc) $)^{5 / 8,11 / 13}$.
A Faculdade de Medicina de Ribeirão Preto tem se preocupado com a avaliação' e com a revi são de sua estrutura curricular e não tem se furtado aos desafios inerentes à implementação de reformas' No entanto, levando-se em conta a multiplicidade de ações necessárias e de acordo com a experiências de implantação do método PBL, anteriormente citadas, seria mais razoável que essa metodologia, se cogita- da a sua aplicação na Faculdade de Medicina de Ribeirão Preto, se restringisse, no início, a determina das unidades de ensino ou segmentos da estrutura cunicular vigente. A avaliação criteriosa dos resulta dos obtidos determinaria a conveniência da utilização mais ampla do método.

\section{AGRADECIMENTOS}

Os autores agradecem as seguintes pessoas, que os acolheram nas respectivas instituições, oferecendo informações e treinamento sobre PBL:

- Howard Barrows, Ann Myers Kelson \& Pamela Moriearty - Southern Illinois School of Medicine;

- Jean Pierre Bernier - Faculté de Médicine, Université de Sherbrooke;

- Minam S. Wetzel - Harvard Medical School;

- Anek Aribarg, Yong Poovarawan \& Boonnart Laisnitsarekul - Faculty of Medicine, Chulalongkorn University.

RODRIGUES M de LV \& FIGUEIREDO JF de C. Problem-based learning. Medicina, Ribeirão Preto, 29: 396402, oct./dec. 1996.

ABSTRACT: Problem-based learning (PBL) is a method in which the learning topics are identified on the basis of the presentation of a real or simulated problem. Once the problem has been presented, the student develops the means to solve it and in this process identifies gaps in his knowledge, which he tries to fill with the help of available instruction resources. The major characteristic of this method is to develop the student's abilities to direct his own learning, to integrate information and to identify and explore new areas.

This method is ideally applicable to student-based self-directed learning. The successful use of the PBL method depends on a series of prerequisites which may affect the form of implantation and the feasibility of the method. Examples of the requirements for the implantation of the PBL method are the need for a space in the curricular schedule for student self-learning, the need to review the role of the teacher in the teaching-learning process, review of the concept of departmental autonomy, changes in student selection criteria, and financial investments in the infrastructure.

Some medical schools use the PBL method in an exclusive manner for every student training, where as others use it for some student groups, and still others use it in segments of the traditional curriculum.

Thus, ii the application of this method is contemplated for the Faculty of Medicine of Ribeirão Preto, it would be advisable first to limit it to certain teaching units or segments of the current curricular structure. A judicious evaluation of the results would then determine the convenience of a broader use of the method.

UNITERMS: Education, Medical. Learning. Faculty, Medical. 


\section{REFERÊNCIAS BIBLIOGRÁFICAS}

1 - BARROWS HS \& TAMBLYM RM. Problem-based learning: an approach to medical education. Springer Publishing, New York, 206 p, 1980.

2 - WETZEL MS. An update on problem based learning at Harvard Medical School. Ann Com Orient Educ 7: 237-247, 1994.

3 - BARROWS HS. The tutorial process. Southern Illinois School of Medicine, Springfield, 63 p, 1988.

4 - BERNIER JP \& DES MARCHAIS JE. Major reform of the program leading to the MD degree at the University of Sherbrooke: a unique experience in pedagogy. Faculté de Médicine, Sherbrooke, 1996, (typescript).

5 - FIGUEIREDO JFC. Relatório. Universidade de São Paulo, Faculdade de Medicina de Ribeirão Preto, 1994 (Visita à Chulalongkorn University).

6 - FIGUEIREDO JFC. Relatório. Universidade de São Paulo, Faculdade de Medicina de Ribeirão Preto, 1995 (Visita à Harvard Medical School).

7 - FIGUEIREDO JFC. Relatório. Universidade de São Paulo, Faculdade de Medicina de Ribeirão Preto, 1996 (Visita à Faculté de Medicine - Université de Sherbrooke).

8 - RODRIGUES MLV. Contribuição de outras áreas do conhecimento ao ensino médico - situação atual. Simpósio: Contribuição de outras áreas do conhecimento ao ensino de graduação em medicina. Anais. Faculdade de Medicina de Ribeirão Preto. Legis Summa, Ribeirão Preto, p.49-55, 1992.

9 - SPAULDING WB. The undergraduate medical curriculum (1969 model): Mc Master University. Can Med Assoc J 100: 659664, 1969.
10- OLIVEIRA JED. Aprendendo e ensinando em medicina. Experiências nacionais e os novos caminhos da Harvard. Rev Assoc Med Bras 37: 127-131, 1991.

11 - GOODENOUGH DA. Changing ground: a medical school lecturer turns to discussion teaching. In: CHRISTENSEN CR; GARVING DA \& SWEET A, eds Education for Judgement: the artistry of discussion leadership. Harvard Business School Press, Boston, p.83-98, 1991.

12- GRAND' MAISON P \& DES MARCHAIS JE. Preparing faculty to teach in a problem based learning curriculum: the Sherbrooke, experience. Faculté de Médicine, Sherbrooke, 1990. (typescript)

13- RODRIGUES MLV \& RODRIGUES CRC. Formação de recursos humanos na área de ensino médico da Faculdade de Medicina de Ribeirão Preto, Universidade de São Paulo. Educ Med Salud 26: 280-284, 1992.

14- TRONCON LEA et ai. Avaliação terminal de competências clínicas dos graduandos em medicina: relato da experiência inicial da Faculdade de Medicina de Ribeirão Preto. Ribeirão Preto, 118 p, 1996.

15- FIGUEIREDO JFC \& RODRIGUES CRC. Estratégias para a reformulação da estrutura curricular da Faculdade de Medicina de Ribeirão Preto na última década e estágio atual do processo. Rev Bras Educ Med 14: 20-23, 1990.

16- FACULDADE DE MEDICINA DE RIBEIRÃO PRETO DA UNIVERSIDADE DE SÃO PAULO. Processo 94.1.1288.17.8, v.3. Estrutura Curricular. FMRP, 1993/1 996.

Recebido para publicação em 23/10/96

Aprovado para publicação em 11/12/96 